\title{
Relationship of activated extracellular signal-regulated kinase $1 / 2$ with lung metastasis in salivary adenoid cystic carcinoma
}

\author{
KAI HU ${ }^{1}$, YE-HUA GAN ${ }^{1}$, SHENG-LIN LI ${ }^{1}$, YAN GAO ${ }^{2}$, \\ DENG-CHENG WU ${ }^{1}$, CUN-YU WANG ${ }^{3}$ and GUANG-YAN YU ${ }^{1}$
}

\begin{abstract}
Departments of ${ }^{1}$ Oral and Maxillofacial Surgery and ${ }^{2}$ Oral Pathology, Peking University
School and Hospital of Stomatology, 22 Zhongguancun Nandajie, Haidian District,

Beijing 100081, P.R. China; ${ }^{3}$ Laboratory of Molecular Signaling, Division of Oral

Biology and Medicine, UCLA School of Dentistry, Los Angeles, CA 90095, USA
\end{abstract}

Received May 2, 2008; Accepted August 22, 2008

DOI: $10.3892 /$ or_00000200

\begin{abstract}
Lung metastasis is one of the important characteristics of salivary adenoid cystic carcinoma (SACC). Although activation of extracellular signal-regulated kinase $1 / 2($ ERK1/2) pathway may be associated with cancer progression in some malignant tumors, its roles in lung metastasis of SACC remains unclear. We examined the expression of activated ERK1/2 in SACC-LM with high lung-metastatic rate and SACC-83 with low lung-metastatic rate, as well as in the tissues from lung-metastatic and nonmetastatic groups of SACC patients. Western blot analysis indicated that SACC-LM exhibited higher expression of activated ERK1/2 than SACC-83. Similarly, immunohistochemistry showed that expression of activated ERK1/2 was detected in $73 \%(8 / 11)$ of the primary tissues from SACC patients with lung metastasis, while only $25 \%$ (3/12) of the primary tissues from SACC patients without lung metastasis $(\mathrm{P}<0.05)$. Furthermore, we examined the effects of U0126, a specific inhibitor of mitogen activated protein kinase kinase (MEK or MAPKK), on migration and invasion in SACCLM cells, showing U0126 not only inactivated ERK1/2, but also inhibited migration and invasion of SACC-LM. The present findings suggested that the elevated expression of activated ERK1/2 may play a role in lung metastasis of salivary adenoid cystic carcinoma.
\end{abstract}

Correspondence to: Professor Guang-Yan Yu, Department of Oral and Maxillofacial Surgery, Peking University School and Hospital of Stomatology, 22 Zhongguancun Nandajie, Haidian District, Beijing 100081, P.R. China

E-mail: gyyu@263.net

Key words: salivary adenoid cystic carcinoma, metastasis, extracellular signal-regulated kinase $1 / 2$, migration, invasion

\section{Introduction}

As one of the most common malignant tumors of salivary glands, salivary adenoid cystic carcinoma (SACC) has some unique characteristics such as neurotropic, infiltrative growth and distant metastasis $(1,2)$. Unlike some other epithelial malignancies with poor prognosis, SACC has a good 5-year survival rate $(\sim 80 \%)$, but 10 - and 20 -year survival rate drops to a lower level $(3,4)$.

Ninety percent of deaths from solid tumors attribute to metastases (5). For SACC patients, distant metastasis is a crucial prognostic factor (6). Previously, we established a human salivary adenoid cystic carcinoma cell line, SACC-83, from a patient suffering from adenoid cystic carcinoma in sublingual gland (7). SACC-83 has a relatively lower lungmetastatic rate that only $33.3 \%$ of the nude mice showed lung metastases three months after injection of the tumor cells into the tail vein of nude mice. Further, a derived cell line SACC-LM with higher lung-metastatic rate was generated from SACC-83 by repeating of culture and injection of the lung metastatic tumor cells into the tail vain of nude mice. The metastatic rate was finally $85.0 \%$ for SACC-LM while only $33.3 \%$ for SACC-83.

Mitogen activated protein kinase (MAPK) pathways are well characterized and believed to have close relationship with metastases, as they regulate many courses involved in cancer progression, such as inducing proteolytic enzymes, initiating several pro-survival genes, maintaining growth, promoting cell migration and invasion (8-10). Accumulating data show that it is significantly activated in many invasive malignant tumors including carcinomas of the breast, colon, kidney and lung (11-14).

Among the three distinct MAPK signaling pathways [extracellular signal-regulated kinase 1/2 (ERK1/2), c-Jun Nterminal kinase/stress-activated protein kinase (JNK/SAPK), and p38], deregulation of ERK1/2 is observed in $\sim 1 / 3$ of all human cancers (15). ERK1/2 activities are often shown to increase in metastatic tumors. For instance, the invasive breast cancer cell lines exhibit constitutively higher expression of activated ERK1/2 compared with the non-invasive breast 
cancer cells and a significant positive association exists between activated ERK1/2 and lymph node metastases of breast cancer (16). In addition, the level of activated ERK1/2 increases with escalating tumor stage in prostate cancer, suggesting activation of ERK1/2 signal pathway is also associated with progression of prostate cancer (17). However, the relationship of ERK1/2 pathway with lung-metastatic rate of SACC has not been defined.

In the present study, we examined the expression of activated ERK1/2 in SACC-83 with lower lung-metastatic rate and SACC-LM with higher lung-metastatic rate, as well as in the lung-metastatic and non-metastatic groups of SACC primary tissues. Furthermore, U0126, a specific inhibitor of mitogen activated protein kinase kinase 1/2 (MEK1/2), was applied to SACC-LM to elucidate the role of activated ERK1/2 in cell migration and invasion.

\section{Materials and methods}

Cell culture. SACC-83 and SACC-LM, previously established in our laboratory, were cultured in RPMI-1640 (Gibco, Grand Island, NY, USA) supplemented with $10 \%$ fetal bovine serum (FBS; Hyclone, Logan, Utah, USA), $100 \mathrm{U} / \mathrm{ml}$ penicillin and $100 \mathrm{U} / \mathrm{ml}$ streptomycin at $37^{\circ} \mathrm{C}$ in a humidified atmosphere of $5 \% \mathrm{CO}_{2}$.

Patients and samples. Formalin-fixed, paraffin-embedded tissue blocks of primary SACC from 1995 to 2007 were retrieved and the corresponding $\mathrm{H} \& \mathrm{E}$ stained sections were reviewed in Department of Oral Pathology, Peking University Hospital of Stomatology. According to WHO classification of salivary gland, 23 SACC samples (9 cribriform type, 9 tubular type and 5 solid type) were selected. Clinical data including gender, age, lesion sites and conditions of lung metastasis were evaluated by reviewing medical charts and pathological records and summarized in Table I. The lung metastatic group was defined as the SACC patients developed lung metastases in 5 years after surgery while the non-metastatic group represented those patients who were free of lung metastases at 5 years.

In vitro wound closure assay. Following serum deprivation overnight, confluent monolayers of SACC cells were scratched with a yellow pipette tip to create wounded areas with distance of 400-600 $\mu \mathrm{m}$. Wounded monolayers were incubated for $24 \mathrm{~h}$ in serum-free medium with vehicle (DMSO) or $10 \mu \mathrm{M}$ U0126 (Promega, Madison, WI, USA). Wounded monolayers were photographed (x40) at 0 and $24 \mathrm{~h}$ after scratching. In vitro wound closure was represented by the decreased distance remaining uncovered by cells. Average rates of wound closure were calculated as the equation of decreased distance/incubated time. Data are presented as mean \pm standard deviation from three independent experiments. To inhibit cell proliferation, parallel experiments were conducted with the addition of $0.5 \mu \mathrm{g} / \mathrm{ml}$ of mitomycin C. Migratory activity was assessed by the rate of wound closure in the presence of mitomycin $\mathrm{C}$, while proliferative activity was determined as the rate of wound closure in the absence of mitomycin $\mathrm{C}$ subtracts the rate of wound closure in the presence of mitomycin $\mathrm{C}$.
Transwell invasion and migration assays. Cell invasion assays were performed using Transwell chambers (Corning Costar, Rochester, NY, USA) containing a polycarbonate membrane filter ( $6.5 \mathrm{~mm}$ diameter, $8 \mu \mathrm{m}$ pore size $)$. In a preliminary cell invasion assays, various loads of extracellar matrix (ECM) gel (20-200 $\mu \mathrm{g} /$ well) were applied. The invaded cells of SACCLM on the bottom surface of the membrane during $12 \mathrm{~h}$ were hardly detected unless $20 \mu \mathrm{g} /$ well ECM gel load was added. Therefore, the membrane was coated with $20 \mu \mathrm{g}$ ECM gel (Sigma-Aldrich, St. Louis, MO, USA) before use. The lower chamber contained 10\% FBS medium. The overnight serumstarved SACC-LM cells were harvested and resuspended in serum-free medium with $0.2 \%$ BSA. After incubation with $10 \mu \mathrm{M}$ U0126 or vehicle for $20 \mathrm{~min}$, the cell suspension was seeded in chambers with $1 \times 10^{5}$ cells/well. After incubation for $12 \mathrm{~h}$ at $37^{\circ} \mathrm{C}$ in $5 \% \mathrm{CO}_{2}$, cells on the top surface of the membrane were wiped off with cotton swabs and the membrane were fixed and stained with crystal violet. Cells on the bottom surface of the membrane were examined with a light microscope at magnification of x200. Cells from 6-10 random fields across three replicate wells were captured for counting and the average number of invaded cells per field was represented activities of cell invasion. For cell migration assays, the same procedure was performed, except that Transwell chambers were not coated with ECM gel.

Western blot analysis. Western blot analysis was performed as previously described (18). Briefly, cells were washed with PBS and lysised with lysis buffer, containing $1 \%$ Nonidet P-40, 5\% sodium deoxycholate, $1 \mathrm{mM}$ phenylmethylsulfonyl fluoride, $100 \mathrm{mM}$ sodium orthovanadate and 1:100 protease inhibitors mixture (Sigma-Aldrich). The protein concentrations were determined using the BCA assay (Pierce, Rockford, IL, USA). Aliquots $(40 \mu \mathrm{g})$ of the protein extracts were subjected to $12 \% \mathrm{SDS}$ polyacrylamide gel electrophoresis and transferred to polyvinylidene difluoride membrane (Millipore, Billerica, MA, USA). The membrane was blocked in the block buffer (5\% nonfat dry milk in TBS-T) for $1 \mathrm{~h}$ and probed with 1/500 dilution of anti-active-ERK1/2 antibodies (Cell Signaling Technology, Beverly, MA, USA) in the same buffer overnight at $4^{\circ} \mathrm{C}$. The membrane was washed extensively with TBS-T and then incubated with horseradish peroxidase-conjugated secondary antibodies against rabbit (Zymed, South San Francisco, CA, USA) for $1 \mathrm{~h}$. After extensive washes with TBS-T, the membrane was visualized using the ECL kit (Applygen Technologies Inc., Beijing, P.R. China). To normalize the protein loading and transfer, the blots were stripped and reprobed with 1/1000 dilution of anti-ERK1/2 antibodies (total ERK1/2; Cell Signaling).

Immunohistochemistry. Paraffin-embedded, multiple tissue sections of $4 \mu \mathrm{m}$ from the $23 \mathrm{SACC}$ samples were placed on Poly-Lysine coated slides and then heated at $60^{\circ} \mathrm{C}$ for $6 \mathrm{~h}$, dried overnight at $37^{\circ} \mathrm{C}$, dewaxed in xylene and rehydrated in graded alcohol. PV-9000 Polymer Detection System for immunohistological staining (Zhongshan Goldenbridge Biotechnology Inc., Beijing, P.R. China) was used. To block endogenous peroxidase activity, sections were placed in $3 \%$ hydrogen peroxide for $10 \mathrm{~min}$ and treated with freshly prepared citrate buffer ( $\mathrm{pH}$ 6.0) for $15 \mathrm{~min}$ in microwave oven 
Table I. Histological subtypes and clinical data.

\begin{tabular}{|c|c|c|c|c|c|}
\hline Case & Tumor type & Gender & Age (years) & Site of lesion & Lung metastasis ${ }^{\mathrm{a}}$ \\
\hline 01 & ACC tubular & Female & 23 & Buccal mucosa & - \\
\hline 02 & ACC tubular & Female & 67 & Sublingual gland & + \\
\hline 03 & ACC tubular & Female & 65 & Sublingual gland & - \\
\hline 04 & ACC tubular & Male & 37 & Mouth floor & - \\
\hline 05 & ACC tubular & Female & 27 & Maxillary & + \\
\hline 06 & ACC tubular & Female & 42 & Submandibular gland & + \\
\hline 07 & ACC tubular & Female & 60 & Palate & - \\
\hline 08 & ACC tubular & Female & 34 & Parotid & + \\
\hline 09 & ACC tubular & Female & 60 & Sublingual gland & + \\
\hline 10 & ACC cribriform & Male & 37 & Zygomatic region & - \\
\hline 11 & ACC cribriform & Male & 60 & Buccal mucosa & - \\
\hline 12 & ACC cribriform & Female & 47 & Mandibular & - \\
\hline 13 & ACC cribriform & Female & 39 & Sublingual gland & - \\
\hline 14 & ACC cribriform & Female & 39 & Base of tongue & - \\
\hline 15 & ACC cribriform & Female & 17 & Sublingual gland & + \\
\hline 16 & ACC cribriform & Female & 51 & Mouth floor & - \\
\hline 17 & ACC cribriform & Male & 55 & Mouth floor & + \\
\hline 18 & ACC cribriform & Male & 64 & Submandibular gland & + \\
\hline 19 & ACC solid & Male & 70 & Submandibular gland & - \\
\hline 20 & ACC solid & Female & 48 & Base of tongue & - \\
\hline 21 & ACC solid & Male & 26 & Palate & + \\
\hline 22 & ACC solid & Male & 37 & Maxillary & + \\
\hline 23 & ACC solid & Female & 37 & Maxillary & + \\
\hline
\end{tabular}

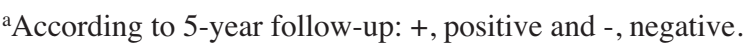

Table II. Status of lung metastasis and activated ERK1/2 (p-ERK1/2) staining in SACC.

\begin{tabular}{lccc}
\hline & \multicolumn{3}{c}{ p-ERK positive } \\
Lung metastasis & $\mathrm{n}$ & $\mathrm{n}(\%)$ & P-value \\
\hline Positive & 11 & $8(73)$ & $0.036^{\mathrm{c}}$ \\
Negative & 12 & $3(25)$ & \\
\hline
\end{tabular}

${ }^{a}$ According to 5-year follow-up. ' Including moderate positive staining

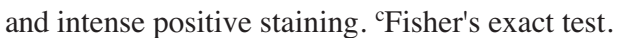

for antigen retrieval. Tissue sections were incubated overnight at $4^{\circ} \mathrm{C}$ with anti-active-ERK1/2 antibodies (1/100 dilution in PBS containing $2 \% \mathrm{BSA}$ ) after an initial incubation with the same antibodies at $37^{\circ} \mathrm{C}$ for $30 \mathrm{~min}$. After the overnight incubation, slides were treated with polymer helper for $20 \mathrm{~min}$ and poly-peroxidase anti-rabbit $\mathrm{IgG}$ for $30 \mathrm{~min}$ at room temperature. Finally, slides were incubated with diaminobenzidine chromogen solution (DAB) for $10 \mathrm{~min}$. Each incubation step was followed by a 2 min PBS-T wash thrice. Finally, sections were counterstained with Mayer's hematoxylin and examined with a light microscope. Negative controls were treated in the same procedure but omitting the primary antibodies. The percentage of immunoreactive cells was estimated by randomly counting 200 tumor cells from
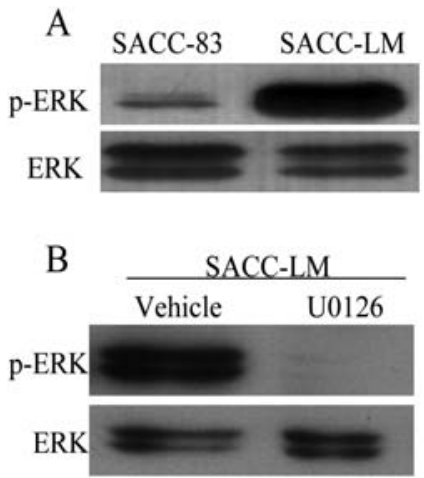

Figure 1. Western blot analysis of activated ERK1/2 in SACC cells. (A) SACC-LM exhibited higher expression of activated ERK1/2 (p-ERK1/2) compared with SACC-83. (B) The activation of ERK1/2 was inhibited in SACC-LM incubated with $10 \mu \mathrm{M}$ U0126 for $10 \mathrm{~min}$.

three different high-power fields (x400) within one specimen. Three semiquantitative classes were used to describe the number of stained cells: negative, $<5 \%$ of the cells; moderate positive, $5-50 \%$ of the cells; intense positive, $>50 \%$ of the cells. Both nuclear and cytoplasmic staining were examined.

Statistical analysis. For studies on SACC cell lines, the results were expressed as mean \pm SD and the significance of the differences between groups was determined by Student's t-test. For studies on SACC tissues in the lung-metastatic and non- 
A

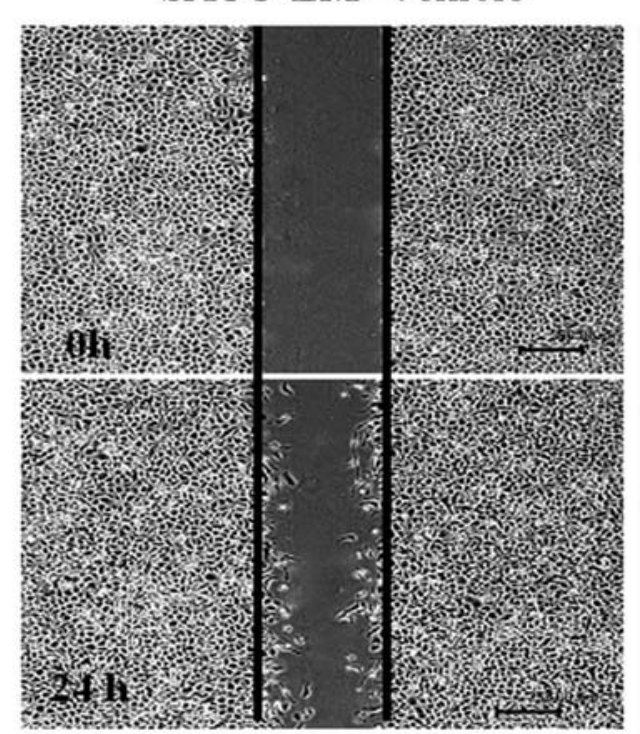

SACC-LM+U0126

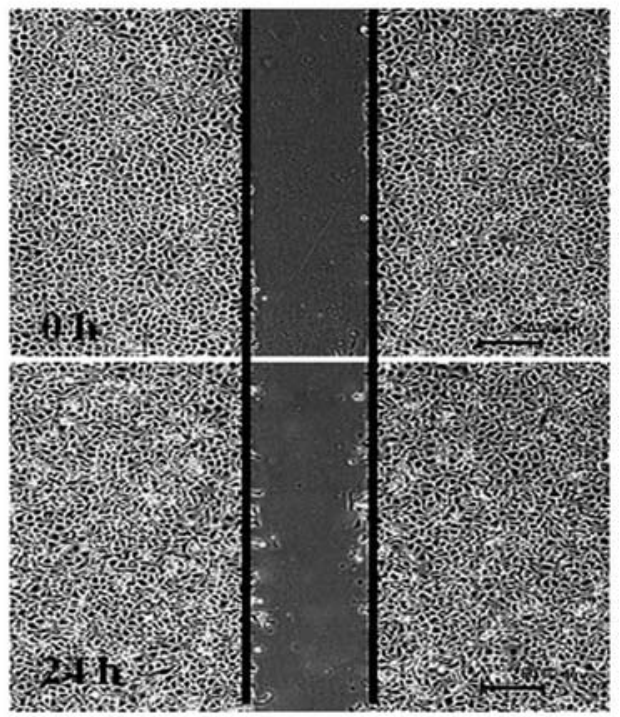

B

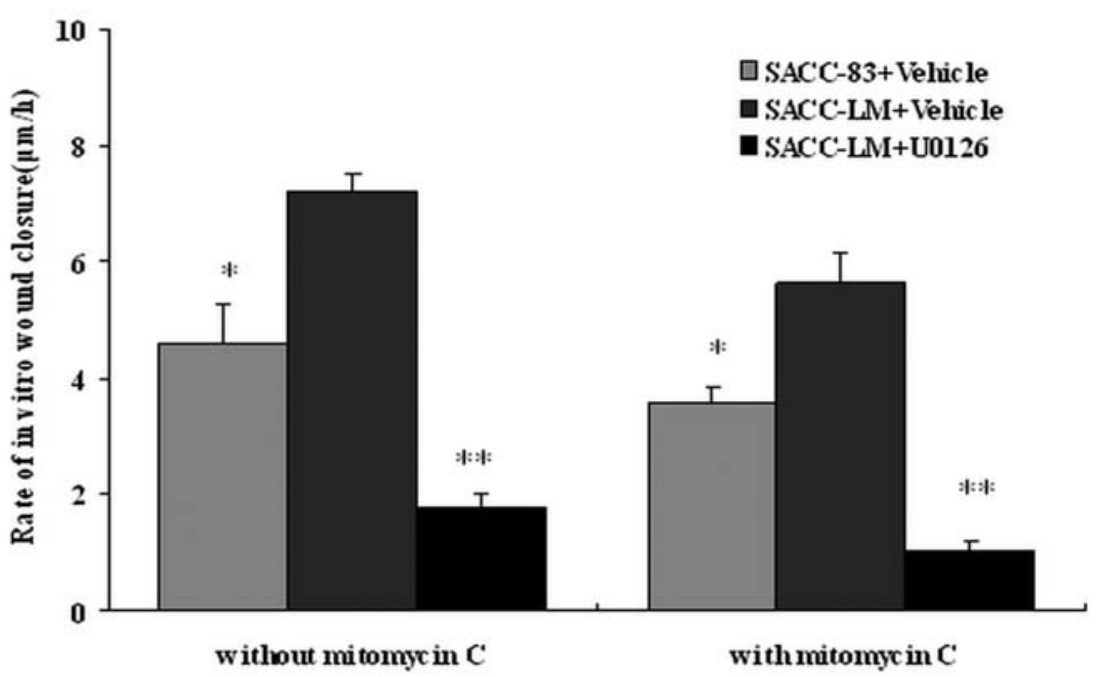

Figure 2. The effects of U0126 on in vitro wound closure in SACC cells. (A) Light microscopy showed that $10 \mu \mathrm{M}$ U0126 delayed in vitro wound closure of SACC-LM cells in the absence of mitomycin C. (B) The rates of wound closure represented the migratory or proliferative activity during 24 h in SACC-83 with vehicle (light gray bars), SACC-LM with vehicle (dark gray bars) and SACC-LM with U0126 (black bars). Results represented mean \pm standard deviation of three separated experiments ( ${ }^{*}$ significantly delayed wound closure in SACC-83+Vehicle vs. SACC-LM+Vehicle, P<0.0005; ${ }^{* *}$ significantly delayed wound closure in SACC-LM+U0126 vs. SACC-LM+Vehicle, $\mathrm{P}<0.0005)$.

metastatic groups, Fisher's exact test was used to test for differences in frequency of detection of active ERK $1 / 2$ between groups. $\mathrm{P}<0.05$ was considered as statistically significant.

\section{Results}

Activated ERK1/2 more highly expressed in SACC-LM than in SACC-83. Western blot showed the expression of activated ERK1/2 in SACC-LM was much higher than that in SACC-83 (Fig. 1A). Moreover, U0126, a specific MEK inhibitor, abolished activation of ERK in SACC-LM at the concentration of $10 \mu \mathrm{M}$ (Fig. 1B).

MEK-specific inhibitor inhibits migration and proliferation of SACC-LM. To determine the effects of activated ERK1/2 on migration of SACC-LM, in vitro wound closure assay and Transwell migration assay were applied. Incubation with $10 \mu \mathrm{M}$ U0126 markedly decreased the wound closure of SACC-LM (Fig. 2A). Migratory and proliferative activity in wound closure assay with or without mitomycin $\mathrm{C}$ (an inhibitor of cell proliferation) were calculated as described in Materials and methods, showing that $10 \mu \mathrm{M}$ U0126 inhibited migratory and proliferative activity of SACC-LM by 81.5 and $54.8 \%$, respectively (Fig. 2B). Besides, incubation with $10 \mu \mathrm{M}$ U0126 decreased Transwell migration of SACC-LM after $12 \mathrm{~h}$ by $38.3 \%(\mathrm{P}<0.0001)$ (Fig. 3A and B), indicating increased activation of ERK1/2 in SACC-LM may contribute to the promoted cell migration.

MEK-specific inhibitor inhibits invasion of SACC-LM. Incubation with $10 \mu \mathrm{M}$ U0126 decreased Transwell invasion 
A

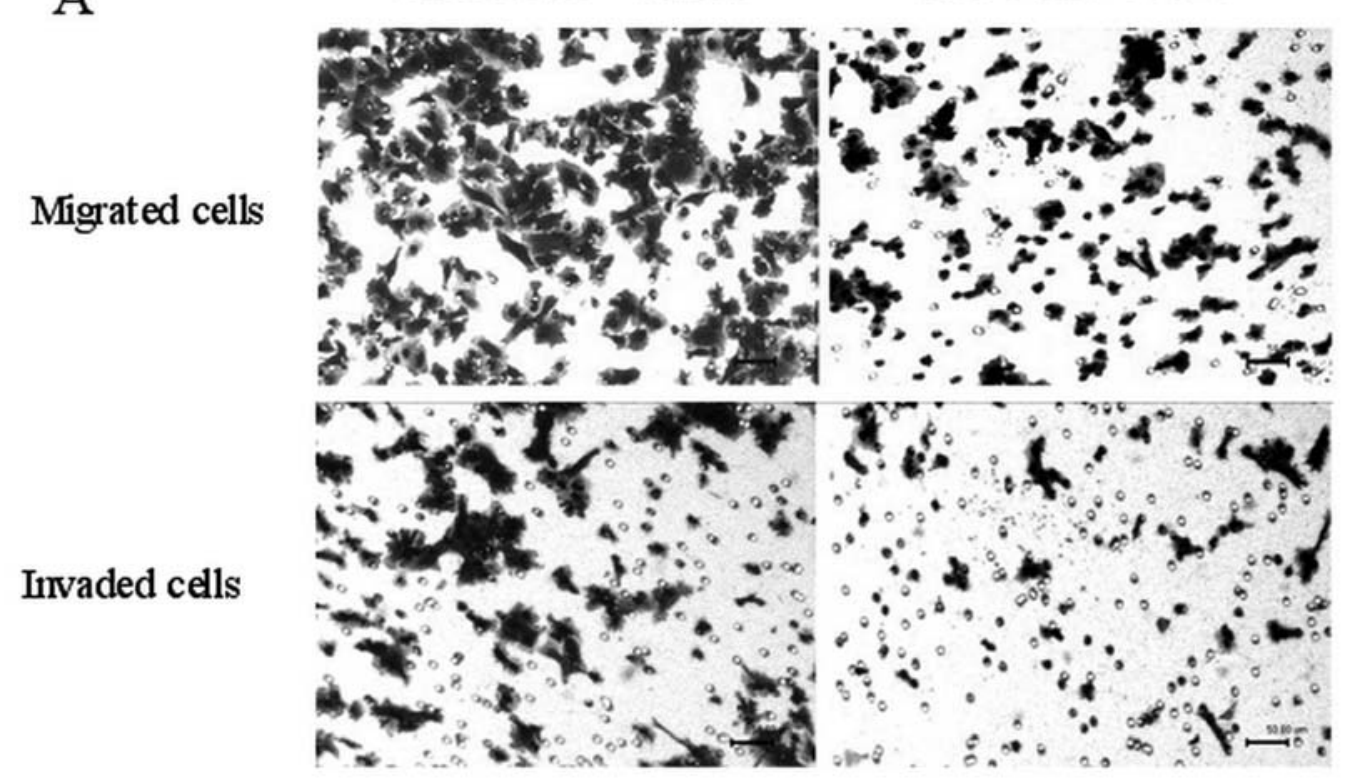

B

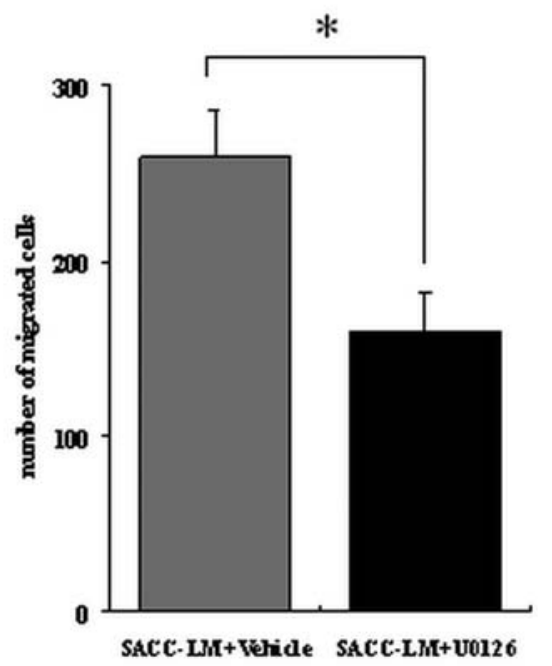

C

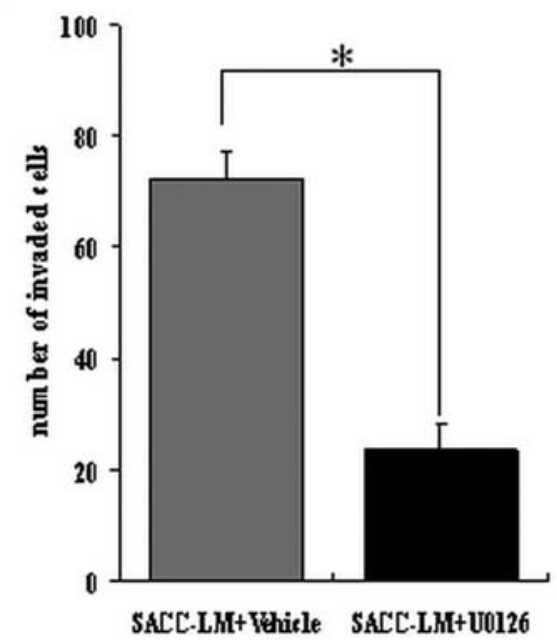

Figure 3. The effects of U0126 on cell migration and invasion of SACC-LM by Transwell assay. (A) Light microscope showed that $10 \mu$ M U0126 decreased the migrated and invaded cells of SACC-LM during $12 \mathrm{~h}$. (B) The average number of migrated cells per field on the bottom surface of the membrane represented the migratory activity. (C) The average number of invaded cells represented the invasive activity. Results in B and $\mathrm{C}$ are mean \pm standard deviation of three experiments carried out at least three times ( ${ }^{*}$ significantly decreased migration vs. SACC-LM+Vehicle, P<0.0001; ${ }^{* *}$ significantly decreased invasion vs. SACC-LM +Vehicle, $\mathrm{P}<0.0001)$.

of SACC-LM during $12 \mathrm{~h}$ by $67.7 \%(\mathrm{P}<0.0001)($ Fig. 3A and $C$ ), suggesting raised expression of activated ERK1/2 may be also involved in promoted cell invasion in SACC-LM.

More frequently expression of activated ERK1/2 in primary tissues of lung-metastatic SACC. Formalin-fixed, paraffinembedded tissues from 12 non-metastatic and 11 lungmetastatic SACC patients were examined immunohistochemically for expression of activated ERK1/2. The results were summarized in Table II. Eight of 11 (73\%) lungmetastatic SACC tissues showed positive staining for activated ERK1/2: three samples showed intense positive staining and five moderate positive staining. However, only three of 12 (25\%) non-metaststic SACC tissues exhibited moderate positive staining for activated ERK1/2, while others were negative. The representative staining for activated ERK1/2 in lung-metastatic and non-metastatic group of cribriform, tubular and solid patterns are shown in Fig. 4.

\section{Discussion}

The present study compared expression of activated ERK1/2 in SACC cell lines and tissues with different lung-metastatic potential and the results indicated that the samples of SACC with high lung-metastatic potential exhibited raised expression of activated ERK1/2.

Although ERK1/2 pathway has been broadly discussed in other malignant tumors, few studies have investigated the role of activated ERK1/2 in malignant tumors of salivary gland. $\mathrm{Hu}$ (19) reported that blockade of ERK1/2 inhibits expression levels of matrix metalloproteinases in adenoid cystic carcinoma cells, indicating ERK1/2 may be associated with 


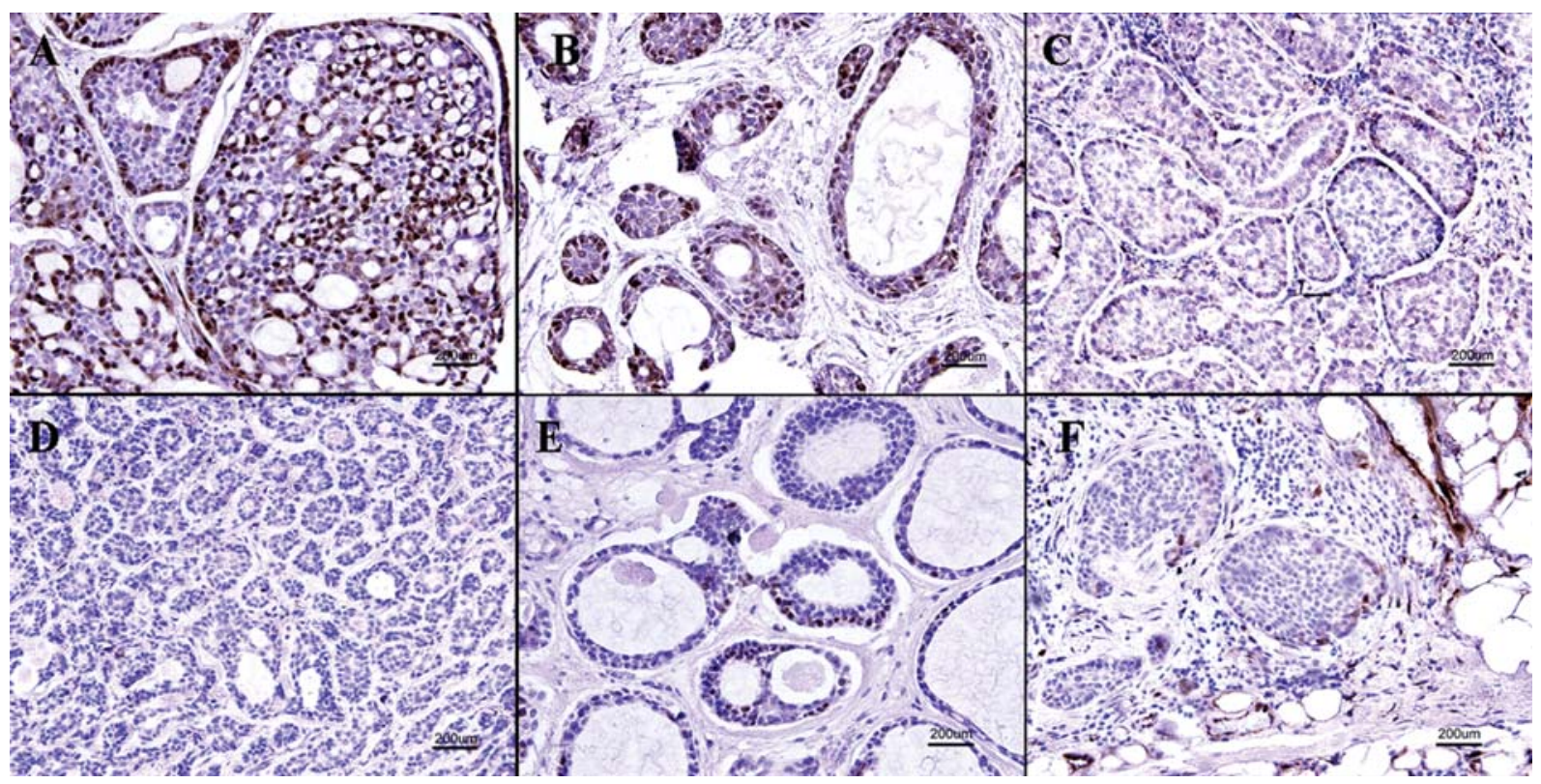

Figure 4. Immunohistochemistry staining of activated ERK1/2 in lung-metastatic and non-metastatic group in tubular (B-E), cribriform (A) and solid (F) pattern of SACC tissues. Representative immunohistochemistry with rabbit anti active-ERK1/2 polyclonal antibodies for lung-metastatic group (A-C) and non-metastatic group (D-F) showed negative staining (D), moderate staining (C, E and F) and intense staining (A and B). Original magnification, $\mathrm{x} 400$.

invasive behaviors of SACC. Handra-Luca et al (20) found that ERK $1 / 2$ pathway is activated in mucoepidermoid carcinoma of salivary gland and suggested it may be positively associated with aggressive behavior as well as proliferative activity. In the present study, we found that activated ERK1/2 was more frequently and more highly expressed in lung-metastatic SACC tissues than in non-metastatic tumors, suggesting activation of ERK1/2 may have a mechanistic role in the metastatic progression of SACC.

A number of studies have demonstrated that ERK/MAPK pathway plays an important role in the regulation of tumor progression, including migration and invasion $(8,9)$. As a fundamental process during normal embryonic development, wound repair, inflammatory response, cell migration also plays an important role in tumor metastasis (21). Activation of ERK1/2 could promote cell migration through triggering the intracellular motility machinery, such as enhancing myosin light-chain kinase (MLCK) activity $(22,23)$ as well as regulating the small G proteins - Rac and Rho (24). Cell invasion, crucial histological marker of metastatic tumors, is characterized with disruption of the integrity of the basement membrane. Through activation of a flock of transcriptional factors including activating protein-1 (AP-1), activated ERK1/2 regulated a number of proteolytic enzymes that degrade the basement membrane, such as matrix metalloproteinases (MMPs) and urokinase plasminogen activator (uPA) $(25,26)$. In some malignant tumors, persistent activation of ERK1/2 was considered to participate in extracellular matrix and basement-membrane degradation that advanced metastases $(25,27)$. Previously, we reported that compared with the lower lung-metastatic rate cell line SACC-83, the higher lungmetastatic cell line SACC-LM exhibited higher ability in cell migration and invasion (28). The present study further displayed that activated ERK1/2 was more highly expressed in SACC-LM than in SACC-83, and inactivation of ERK1/2 led to a significant inhibition of cell migration and invasion in SACC-LM. These findings suggested the markedly increased expression of activated ERK $1 / 2$ could be partially the cause for the increased cell migration and invasion of SACC-LM.

There are several possible mechanisms to induce activation of ERK/MAPK signaling. Growth factors and adhesion molecules often mediate receptor tyrosine kinase pathways and subsequent activation of ERK1/2 (29). Previously, we found that the mRNA expression of epiregulin, a ligand of EGF receptor, was much higher in SACC-LM than in SACC-83 (28). As a pan-EGFR ligand, epiregulin not only elicits a more potent mitogenic signal than epidermal growth factor (30), but also participates in pulmonary metastatases in some invasive cancers $(31,32)$. It has been reported that compared with EGF or TGF- $\alpha$, epiregulin was more potent and effective in inducing significantly greater ERK $1 / 2$ activation (33). In addition, we also found that epiregulin induced great transient activation of ERK1/2 in SACC-83 (28), suggesting that the elevated ERK1/2 activity in SACC-LM may be partly due to the increased expression of epiregulin.

In summary, significantly increased expression of activated ERK1/2 were found in SACC cell lines and tissues with higher lung-metastatic capability. Elevated activation of ERK1/2 may contribute to promoting cell migration and invasion in SACC cell line with higher lung-metastasis rate. The present findings suggest that increased expression of activated ERK1/2 could be associated with lung metastasis in salivary adenoid cystic carcinoma.

\section{Acknowledgements}

We gratefully acknowledge Dr Xin Peng from Department of Oral and Maxillofacial Surgery, Peking University School and Hospital of Stomatology and Dr Xin Li from the Department of Oral Pathology, Peking University School and Hospital of Stomatology, for providing clinical information of SACC 
patients. This study was supported by the National Natural Science Foundation of P.R. China (Grant No. 30572050) and the National Science Foundation of Beijing, P.R. China (Grant No. 7062069).

\section{References}

1. Szanto PA, Luna MA, Tortoledo E and White RA: Histologic grading of adenoid cystic carcinoma of the salivary glands. Cancer 54: 1062-1069, 1984.

2. Zhang J, Peng B and Chen X: Expressions of nuclear factor kappa B, inducible nitric oxide synthase, and vascular endothelial growth factor in adenoid cystic carcinoma of salivary glands: correlations with the angiogenesis and clinical outcome. Clin Cancer Res 11: 7334-7343, 2005.

3. Huang M, Ma D, Sun K, Yu G, Guo C and Gao F: Factors influencing survival rate in adenoid cystic carcinoma of the salivary glands. Int J Oral Maxillofac Surg 26: 435-439, 1997.

4. Sung MW, Kim KH, Kim JW, et al: Clinicopathologic predictors and impact of distant metastasis from adenoid cystic carcinoma of the head and neck. Arch Otolaryngol Head Neck Surg 129: 1193-1197, 2003.

5. Gupta GP and Massague J: Cancer metastasis: Building a framework. Cell 127: 679-695, 2006.

6. Rapidis AD, Givalos N, Gakiopoulou H, et al: Adenoid cystic carcinoma of the head and neck. Clinicopathological analysis of 23 patients and review of the literature. Oral Oncol 41: 328-335, 2005.

7. Li SL, Liu XP and Zhang KH: Establishment of a human cancer cell line from adenoid cystic carcinoma of the minor salivary gland. Zhonghua Kou Qiang Yi Xue Za Zhi 25: 29-31, 1990.

8. Reddy KB, Nabha SM and Atanaskova N: Role of MAP kinase in tumor progression and invasion. Cancer Metastasis Rev 22: 395-403, 2003.

9. Krueger JS, Keshamouni VG, Atanaskova N and Reddy KB: Temporal and quantitative regulation of mitogen-activated protein kinase (MAPK) modulates cell motility and invasion. Oncogene 20: 4209-4218, 2001.

10. Giehl K: Oncogenic Ras in tumour progression and metastasis. Biol Chem 386: 193-205, 2005.

11. Hoshino R, Chatani Y, Yamori T, et al: Constitutive activation of the 41-/43-kDa mitogen-activated protein kinase signaling pathway in human tumors. Oncogene 18: 813-822, 1999.

12. Sivaraman VS, Wang H, Nuovo GJ and Malbon CC: Hyperexpression of mitogen-activated protein kinase in human breast cancer. J Clin Invest 99: 1478-1483, 1997.

13. Salh B, Marotta A, Matthewson C, Ahluwalia M, Flint J, Owen D and Pelech S: Investigation of the Mek-MAP kinase-Rsk pathway in human breast cancer. Anticancer Res 19: 731-740, 1999.

14. Mueller H, Flury N, Eppenberger-Castori S, Kueng W, David F and Eppenberger U: Potential prognostic value of mitogenactivated protein kinase activity for disease-free survival of primary breast cancer patients. Int J Cancer 89: 384-388, 2000

15. Mercer KE and Pritchard CA: Raf proteins and cancer: B-Raf is identified as a mutational target. Biochim Biophys Acta Rev Cancer 1653: 25-40, 2003.

16. Adeyinka A, Nui Y, Cherlet T, Snell L, Watson PH and Murphy LC: Activated mitogen-activated protein kinase expression during human breast tumorigenesis and breast cancer progression. Clin Cancer Res 8: 1747-1753, 2002.
17. Gioeli D, Mandell JW, Petroni GR, Frierson HF and Weber MJ: Activation of mitogen-activated protein kinase associated with prostate cancer progression. Cancer Res 59: 279-284, 1999.

18. Zeng QH, Chen SQ, You ZB, Yang F, Carey TE, Saims D and Wang CY: Hepatocyte growth factor inhibits anoikis in head and neck squamous cell carcinoma cells by activation of ERK and Akt signaling independent of NF kappa B. J Biol Chem 277: 25203-25208, 2002.

19. Hu JA: Blockade of the ERK pathway down-regulation MMPS in adenoid cystic carcinoma. J Oral Pathol Med 35: 428, 2006.

20. Handra-Luca A, Bilal H, Bertrand JC and Fouret P: Extra-cellular signal-regulated ERK-1/ERK-2 pathway activation in human salivary gland mucoepidermoid carcinoma - Association to aggressive tumor behavior and tumor cell proliferation. Am J Pathol 163: 957-967, 2003.

21. Lauffenburger DA and Horwitz AF: Cell migration: A physically integrated molecular process. Cell 84: 359-369, 1996.

22. Hartmann G, Weidner KM, Schwarz H and Birchmeier W: The motility signal of scatter factor/hepatocyte growth factor mediated through the receptor tyrosine kinase met requires intracellular action of Ras. J Biol Chem 269: 21936-21939, 1994.

23. Klemke RL, Yebra M, Bayna EM and Cheresh DA: Receptor tyrosine kinase signaling required for integrin alpha $\mathrm{v}$ beta 5directed cell motility but not adhesion on vitronectin. J Cell Biol 127: 859-866, 1994.

24. Vial E, Sahai E and Marshall CJ: ERK-MAPK signaling coordinately regulates activity of Rac1 and RhoA for tumor cell motility. Cancer Cell 4: 67-79, 2003.

25. Overall CM and Lopez-Otin C: Strategies for mmp inhibition in cancer: innovations for the post-trial era. Nat Rev Cancer 2: 657-672, 2002.

26. Ree AH, Bjornland K, Brunner N, Johansen HT, Pedersen KB, Aasen AO and Fodstad O: Regulation of tissue-degrading factors and in vitro invasiveness in progression of breast cancer cells. Clin Exp Metastasis 16: 205-215, 1998.

27. Egeblad M and Werb Z: New functions for the matrix metalloproteinases in cancer progression. Nat Rev Cancer 2: 161-174, 2002.

28. Hu K, Li SL, Gan YH, Wang CY and Yu GY: Epiregulin promotes migration and invasion of salivary adenoid cystic carcinoma cell line SACC-83 through activation of ERK and Akt. Oral Oncol (In press).

29. Katz M, Amit I and Yarden Y: Regulation of MAPKs by growth factors and receptor tyrosine kinases. Biochim Biophys Acta Mol Cell Res 1773: 1161-1176, 2007.

30. Shelly M, Pinkas-Kramarski R, Guarino BC, et al: Epiregulin is a potent pan-ErbB ligand that preferentially activates heterodimeric receptor complexes. J Biol Chem 273: 10496-10505, 1998.

31. Nicholson BE, Frierson HF, Conaway MR, Seraj JM, Harding MA, Hampton GM and Theodorescu D: Profiling the evolution of human metastatic bladder cancer. Cancer Res 64: 7813-7821, 2004.

32. Gupta GP, Nguyen DX, Chiang AC, et al: Mediators of vascular remodelling co-opted for sequential steps in lung metastasis. Nature 446: 765-770, 2007.

33. Draper BK, Komurasaki T, Davidson MK and Nanney LB: Epiregulin is more potent than EGF or TGFalpha in promoting in vitro wound closure due to enhanced ERK/MAPK activation. J Cell Biochem 89: 1126-1137, 2003. 